M. Hitsuda

Nagoya Math. J.

Vol. 52 (1973), 39-46

\title{
MULTIPLICITY OF SOME CLASSES OF GAUSSIAN PROCESSES
}

\section{MASUYUKI HITSUDA}

\section{Introduction.}

The aim of this paper is to discuss the multiplicity of the sum of two independent Gaussian processes

$$
x(t)=x_{1}(t)+x_{2}(t),
$$

where $x_{1}(t)$ is a Wiener process and $x_{2}(t)=F(t) \int_{0}^{t} G(s) d B_{2}(s)$ is a simple Markov process.

Intuitively speaking, the process $x(t)$ may be considered as a received message disturbed by a noise $x_{1}(t)$ which can be taken to be a Wiener process. The problem discussed here is asking whether we can separate the message and the noise in the sense of the multiplicity introduced in the theory of canonical representation.

The concept of multiplicity was established by $T$. Hida [2] and $\mathrm{H}$. Cramér [1], which treated the so-called canonical representation of Gaussian processes proposed by P. Lévy [4]. Since then many authors have discussed the problem: One of the main interests has been a problem to find out the multiplicity and the associated measure for a given Gaussian process. In his earlier paper, the author of the present paper has proved that Gaussian processes $x(t)$ equivalent to a Wiener process has the canonical representation of multiplicity one [3], where the $x(t)$ is necessarily expressed as in (1.1), but $x_{2}(t)$ is measurable with respect to the Wiener process $x_{1}(t)$. Recently, T. Н. Сирая [5] has discussed the multiplicity of the sum of two processes, using the reproducing kernel Hilbert space technique, which was formulated in [2]. In this paper, we can see the multiplicity and the basic measure more definitely in a rather restricted class of Gaussian processes.

Received May 15, 1973. 
The result is this: When $G(s)$ is positive and continuous in the formula (1.1), the multiplicity and the basic measures depend upon $F(t)$. (I) When $F^{\prime}(t)$ belongs to $L^{2}$, the basic measure is Lebesgue and the multiplicity is one. (II) When $F^{\prime}(t)$ does not belong to $L^{2}(l, m)$ for any open interval $(l, m)$, the multiplicity is two and basic measures are Lebesgue. (III) When $F(t)$ is of unbounded variation in any open interval, the same result as in (II) holds. The proof heavily depends upon the previous result [3].

In the last section, applications of our theorem shall be given. We shall construct a process $x(t)$ of the type (1.1), whose basic measure has a singular part. And we shall show the existence of an $N$-ple Markov Gaussian process with the multiplicity $N>1$, to illustrate the corollary.

\section{Main Theorem.}

We first give a lemma describing the relation between a Gaussian process and the corresponding reproducing kernel Hilbert space (RKHS).

LEMMA. Let $x(t)(t \geq 0)$ be a Gaussian process with $E x(t)=0$, and let $\mathfrak{S}_{t}$ be a Hilbert space with the reproducing kernel $\Gamma(s, u)=E(x(s) x(u))$, $s, u \leq t$.

(I) If a Gaussian process $x(t)$ has a canonical representation

$$
x(t)=\int_{0}^{t} F(t, u) d B(u),
$$

where $B(t)$ is a Wiener process, then

$$
\mathfrak{S}_{t}=\left\{a ; a(u)=\int_{0}^{u} F(u, v) \alpha(v) d v, \quad 0 \leq u \leq t, \alpha \in L^{2}[0, t]\right\} .
$$

(II) If a Gaussian process $x(t)$ is a sum of two independent canonical processes: $x(t)=x_{1}(t)+x_{2}(t)$, where

$$
x_{1}(t)=\int_{0}^{t} F_{1}(t, u) d B_{1}(u), \quad x_{2}(t)=\int_{0}^{t} F_{2}(t, u) d B_{2}(u)
$$

and $B_{i}(t)^{\prime} s(i=1,2)$ are mutually independent Wiener processes, then

$$
\mathscr{S}_{t}=\left\{a_{1}+a_{2} ; a_{1} \in \mathscr{S}_{t}^{1}, a_{2} \in \mathscr{S}_{t}^{2}\right\},
$$

where $\mathfrak{S}_{t}^{1}$ and $\mathfrak{S}_{t}^{2}$ are $R$ KHS's corresponding to $x_{1}(t)$ and $x_{2}(t)$ respectively. 
(III) In the case (II), if $\mathfrak{S}_{t}^{1} \cap \mathfrak{S}_{t}^{2}=\{0\}$ for each $t$, then the multiplicity of $x(t)$ is two and (1.1) is the canonical representation.

Proofs of (I) and (II) are included in $\S$ I of [2], and (III) is proved in $[\dot{5}]$, so they are omitted.

Let $B_{i}(t) \quad(i=1,2)$ be Wiener processes which are mutually independent. And let

$$
x(t)=x_{1}(t)+x_{2}(t)=B_{1}(t)+F(t) B_{2}(t) \quad(t \geq 0) .
$$

We note that the representation $F(t) B(t)$, where $B(t)$ is a Wiener process, is canonical in the sense of Hida [2], and note that it is a Gaussian Markov process.

The assertion stated below is valid in the case of $x_{2}(t)=$ $F(t) \int_{0}^{t} G(s) d B_{2}(s)$ for a positive and continuous function $G(s)$. But, essentially, we may only discuss the case $x_{2}(t)=F(t) B_{2}(t)$, because the proof of the theorem can be rewritten in an easy manner.

THEOREM. (I) If $F(t)$ in (2.1) is absolutely continuous and if the Radon-Nikodym derivative $F^{\prime}(t)$ belongs to $L^{2}\left[0, t_{0}\right]$ for any $t_{0} \geq 0$, the multiplicity of the Gaussian process $x(t)$ is one and the basic measure is the Lebesgue measure.

(II) If $F(t)$ in (2.1) is absolutely continuous but $F^{\prime}(t)$ does not belong to $L^{2}(l, m)$ for any open interval $(l, m)$, the multiplicity of $x(t)$ is two and the basic measures are Lebesgue measures.

(III) If $F(t)$ in (2.1) is of unbounded variation in everywhere, the multiplicity of $x(t)$ is two and the basic measures are Lebesgue measures.

Remark. In cases which are not stated in Theorem, the circumstance is more complicated. Under some condition on $F(t)$, the basic measure has a singular part with respect to the Lebesgue measure as we shall see such an example in the following section.

Proof of (I). We can rewrite (2.1) in the form

$$
x(t)=B_{1}(t)+\int_{0}^{t} F(s) d B_{2}(s)+\int_{0}^{t} F^{\prime}(s) B_{2}(s) d s .
$$

Then $\tilde{B}(t)=B_{1}(t)+\int_{0}^{t} F(s) d B_{2}(s)$ is a Gaussian martingale with respect to the $\sigma$-field $\mathfrak{B}_{1}(t) \vee \mathfrak{B}_{2}(t)=\sigma\left(B_{1}(s) ; s \leq t\right) \vee \sigma\left(B_{2}(s) ; s \leq t\right)$, and the cor- 
responding increasing process is deterministic and is expressed as

$$
A(t)=\int_{0}^{t}\left(1+F(s)^{2}\right) d s .
$$

On the other hand, the integrand of the third component $F^{\prime}(s) B_{2}(s)$ of the right hand side of (2.2) is $\mathfrak{B}_{1}(s) \vee \mathfrak{B}_{2}(s)$-measurable and we get

$$
\begin{aligned}
& E\left[\int_{0}^{t}\left(F^{\prime}(s) B_{2}(s)\right)^{2} d s\right] \\
& \quad=E\left[\int_{0}^{t}\left(\frac{F^{\prime}(s) B_{2}(s)}{1+F(s)^{2}}\right)^{2}\left(1+F(s)^{2}\right) d s\right] \\
& \quad=\int_{0}^{t} \frac{F^{\prime}(s)^{2} s}{1+F(s)^{2}} d A(s)<\infty .
\end{aligned}
$$

Therefore $x(t)$ is equivalent to (2.2), so the multiplicity of $x(t)$ is one by the result of [3].

Proof of (II). The RKHS corresponding to $x_{1}(t)=B_{1}(t)$ and $x_{2}(t)$ $=F(t) B_{2}(t)$ are

$$
\mathfrak{S}_{c}^{1}=\left\{a(\cdot) ; a(s)=\int_{0}^{s} \alpha(u) d u \quad(s \leq t), \quad \alpha \in L^{2}[0, t]\right\}
$$

and

$$
\mathfrak{S}_{t}^{2}=\left\{F(\cdot) a(\cdot) ; a(s)=\int_{0}^{s} \alpha(u) d u \quad(s \leq t), \quad \alpha \in L^{2}[0, t]\right\},
$$

respectively. In order to prove that the multiplicity of $x(t)$ is two, it is enough to show that

$$
\mathfrak{S}_{t}^{1} \cap \mathfrak{S}_{t}^{2}=\{0\}
$$

by Lemma. Let $a \in \mathfrak{S}_{t}^{1} \cap \mathfrak{S}_{t}^{2}$. Then we can write $a$ as

$$
a(s)=\int_{0}^{s} \alpha_{1}(u) d u=F(s) \int_{0}^{s} \alpha_{2}(u) d u \quad \alpha_{1}, \alpha_{2} \in L^{2}[0, t] .
$$

Differentiating both sides of (2.3), we get

$$
\frac{d a(u)}{d u}=\alpha_{1}(u)=F^{\prime}(u) \int_{0}^{u} \alpha_{2}(v) d v+F(u) \alpha_{2}(u) \text {. }
$$

Therefore $F^{\prime}(u) \int_{0}^{u} \alpha_{2}(v) d v$ must belong to $L^{2}(l, m)$ for any open interval $(l, m) \subset[0, t]$. So 


$$
a_{2}(u)=\int_{0}^{u} \alpha_{2}(v) d v=0 \quad \text { for almost all } u \in[0, t]
$$

and $a_{2}(u)=0 u \in[0, t]$, because of the continuity of $a_{2}(u)$. Therefore $\alpha_{2}(u)=0$, which implies $a=0$.

Proof of (III). As in the proof of (II), we only show the equality $\mathfrak{S}_{t}^{1} \cap \mathfrak{S}_{t}^{2}=\{0\} . \quad$ Let $a \in \mathfrak{S}_{t}^{1} \cap \mathfrak{S}_{\mathrm{c}}^{2}$. Then

$$
a(s)=F(s) \int_{0}^{s} \alpha_{2}(u) d u
$$

must be absolutely continuous, so $\int_{0}^{s} \alpha_{2}(u) d u=0$. Because, if $a_{2}(s)=$ $\int_{0}^{s} \alpha_{2}(u) d u \neq 0$ for some $s \in[0, t]$, then $F(t)=\frac{F(t) a_{2}(t)}{a_{2}(t)}$ must be of bounded variation in some neighbourhood of $s$.

Thus the proof of Theorem is completed.

COROLlary (I) If $F(t)$ in (2.1) satisfies the condition of Theorem (I), then the process

$$
y(t)=B_{1}(t)+F(t) B_{2}(t)+\cdots+F(t)^{N-1} B_{N}(t),
$$

where $B_{i}(t)$ 's $(i=1,2, \cdots, N)$ are mutually independent Wiener processes, is of multiplicity one and the basic measure is Lebesgue.

(II) If $F(t)$ satisfies the condition of Theorem (II), then the process $y(t)$ defined by (2.3) is of multiplicity $N$.

Proof of (I) is almost same as in Theorem (I), so it is omitted.

Proof of (II). It is enough to show that the RKHS corresponding to the process $y(t)$ is

$$
\mathfrak{S}_{t}=\mathfrak{S}_{t}^{1} \oplus \mathfrak{S}_{t}^{2} \oplus \cdots \oplus \mathfrak{S}_{t}^{N}
$$

where $\mathfrak{S}_{\mathrm{c} t}^{i}=\left\{F(\cdot)^{i-1} a(\cdot) ; a(s)=\int_{0}^{s^{\prime}} \alpha(u) d u, \alpha \in L^{2}[0, t]\right\} \quad$ (cf. [2]). Suppose that

$$
a_{1}(s)+F(s) a_{2}(s)+\cdots+F(s)^{N-1} a_{N}(s)=0, \quad a_{i} \in \mathfrak{S}_{t}^{i} .
$$

Differentiating the left hand side of (2.4), we get 


$$
\begin{aligned}
a_{1}^{\prime}(s) & +F(s) a_{2}^{\prime}(s)+\cdots+F(s)^{N-1} a_{N}^{\prime}(s) \\
& +F^{\prime}(s)\left(a_{2}(s)+\cdots+(N-1) F(s)^{N-2} a_{N}(s)\right)=0 .
\end{aligned}
$$

As $F^{\prime}(s)$ does not belong to $L^{2}(l, m)$ for any open interval $:(l, m)$, we must have

$$
a_{2}(s)+\cdots+(N-1) F(s)^{N-2} a_{N}(s)=0 .
$$

So, by induction, we can conclude the desired result.

Remark. In case that $F(t)$ is of unbounded variation everywhere, it is conjectured that the same result as in Corollary (II) will hold, but it is not proved yet.

\section{Concluding Remarks.}

This section is devoted to state some further developments which follow from our main Theorem.

1. In case that the RKHS's $\mathfrak{S}_{t}^{1}$ and $\mathscr{S}_{t}^{2}$, corresponding to $B_{1}(t)$ and $F(t) B_{2}(t)$ respectively, have some nontrivial intersection, the circumstance is more complicated. Here we give an example for illustration.

EXAMPle 1. If $F(t)$ is decomposed in the form

$$
F(t)=\int_{0}^{t} f_{1}(s) d s+F_{2}(t)
$$

where $f_{1} \in L^{2}[0, \infty)$ and $F_{2}(t)$ is a step function. Then the process

$$
x(t)=B_{1}(t)+F(t) B_{2}(t)
$$

has the representation of the multiplicity one. But the basic measure is $m(d u)+n(d u)$, where $m(d u)$ is the Lebesgue measure and $n(d u)$ is the point measure induced by $F_{2}(t)$.

2. Double Markov Gaussian process with multiplicity two.

Hida [2] defined the $N$-ple Markov Gaussian processes and studied such processes with multiplicity one. Here we can give an example of a double Markov Gaussian process with the multiplicity two, two basic measures of which are Lebesgue. 
EXAMPLE 2. We can construct a function $F(t)$ satisfying the following conditions :

(i ) $F(t)$ is absolutely continuous,

(ii) the Radon-Nikodym derivative $F^{\prime}(t)$ is strictly positive, and

(iii) it does not belong to $L^{2}(l, m)$ for any open interval $(l, m) \subset$ $[0, \infty)$, but does belong to $L^{1}[0, t]$ for any $t>0$. Using such an $F(t)$, put

$$
x(t)=B_{1}(t)+F(t) B_{2}(t) .
$$

Then the process $x(t)$ is an example of the desired processes. In fact, by Theorem (II), $x(t)$ has multiplicity two. And we have

$$
\begin{aligned}
E\left[x(t) \mid \mathfrak{X}\left(t_{0}\right)\right] & =E\left[x(t) \mid \mathfrak{B}_{1}\left(t_{0}\right) \vee \mathfrak{B}_{2}\left(t_{0}\right)\right] \\
& =B_{1}\left(t_{0}\right)+F(t) B_{2}\left(t_{0}\right) \quad\left(t_{0}<t\right),
\end{aligned}
$$

where $\mathfrak{X}\left(t_{0}\right), \mathfrak{B}_{1}\left(t_{0}\right)$ and $\mathfrak{B}_{2}\left(t_{0}\right)$, are $\sigma$-algebras generated by $x(s), B_{1}(s)$ and $B_{2}(s) \quad\left(s \leq t_{0}\right)$, respectively. For any $t_{0}<t_{1}<t_{2}, \quad E\left[x\left(t_{1}\right) \mid \mathfrak{X}\left(t_{0}\right)\right]$ and $E\left[x\left(t_{2}\right) \mid \mathfrak{X}\left(t_{0}\right)\right]$ are linearly independent, because $F(t)$ is strictly increasing. On the other hand, for $t_{0}<t_{1}<t_{2}<t_{3}$, the system

$$
\left\{E\left[x\left(t_{i}\right) \mid \mathfrak{X}\left(t_{0}\right)\right] ; i=1,2,3\right\}
$$

is linearly dependent. So $x(t)$ is of multiplicity two, and the representation (3.2) is canonical. Of course the basic measures are Lebesgue.

Furthermore, making use of the idea of this example, we can construct an $N$-ple Markov Gaussian process with multiplicity $N$ and with Lebesgue measures as its basic measures.

EXAMPLE 3. Let $F(t)$ be as in Example 2. Then the Gaussian process

$$
x(t)=B_{1}(t)+F(t) B_{2}(t)+F(t)^{2} B_{3}(t)+\cdots+F(t)^{N-1} B_{N}(t),
$$

where $B_{i}(t)$ 's $(i=1,2, \cdots, N)$ are mutually independent Wiener processes, is an $N$-ple Markov Gaussian process with the multiplicity $N$. This result follows from Corollary (II).

\section{REFERENCES}

[1] H. Cramér, On some classes of non-stationary stochastic processes, Proc. 4th Berkeley Symp. Math. Stat. and Prob., (1961), 57-77. 
[2] T. Hida, Canonical representations of Gaussian processes and their applications, Mem. Coll. Sci. Univ. Kyoto, 33 (1960), 109-155.

[ 3 ] M. Hitsuda, Representation of Gaussian processes equivalent to Wiener process, Osaka J. Math., 5 (1968), 299-312.

[4] P. Lévy, A special problem of Brownian motion and a general theory of Gaussian random functions, Proc. 3rd Berkeley Symp. Math. Stat. and Prob., (1956), 133-175.

[ 5 ] Т. Н. Сирая, О канонических представлениях случайных процессов кратностеи один и два, теория вероят. и ее прим., 18, (1973), 155-160.

Nagoya Institute of Technology 